\title{
Contribuição diagnnóstica da avaliação eletrofisiológica visual em pacientes atendidos em hospital universitário
}

\author{
Diagnostic contribution of visual electrophysiologic assessment in patients from a \\ universityhospital
}

\author{
Paula Yuri Sacai ${ }^{1}$ \\ Adriana Berezovsky ${ }^{2}$ \\ Sérgio Costa Fantini ${ }^{3}$ \\ Solange Rios Salomão ${ }^{4}$
}

Trabalho realizado no Laboratório de Eletrofisiologia Visual Clínica da Universidade Federal de São Paulo UNIFESP.

${ }^{1}$ Tecnóloga Oftálmica

${ }^{2}$ Doutora em Ciências Visuais pela Universidade Federal de São Paulo - UNIFESP

3 Médico Oftalmologista pela Universidade Federal de São Paulo - UNIFESP

${ }^{4}$ Professora Adjunta, Chefe do Laboratório de Eletrofisiologia Visual Clínica da Universidade Federal de São Paulo - UNIFESP

Endereço para correspondência: R. Botucatu 822 São Paulo (SP) CEP 04013-062

E-mail:psacai@oftalmo.epm.br

Recebido para publicação em 07.12.2001

Aceito para publicação em 20.06.2002

\begin{tabular}{|c|}
\hline RESUMO \\
\hline 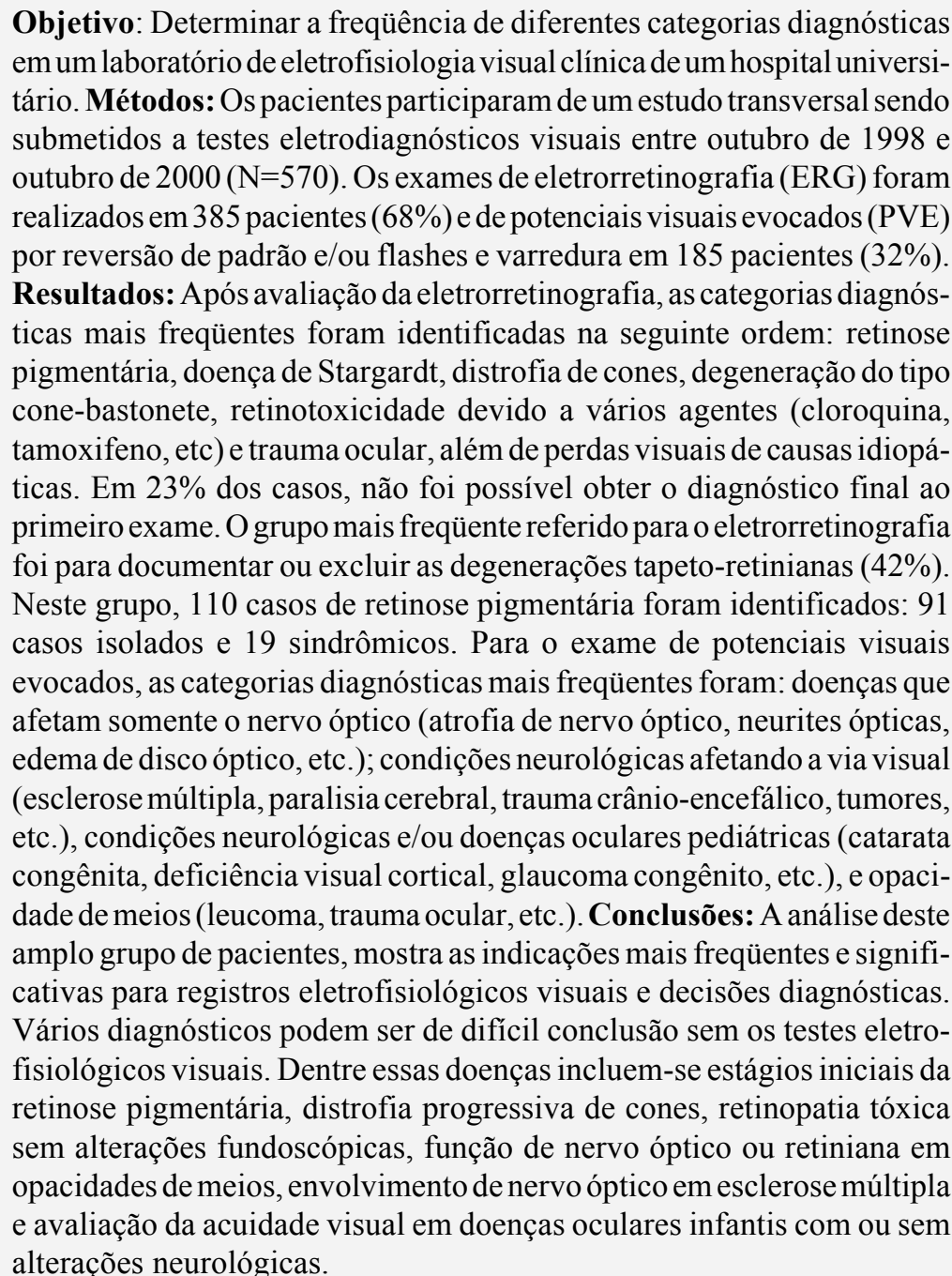 \\
\hline
\end{tabular}

Descritores: Eletrorretinografia; Retina/fisiopatologia; Potenciais evocados visuais; Visão/ fisiologia; Eletrofisiologia/métodos; Testes visuais; Doenças retinianas/diagnóstico; Retinite pigmentosa/diagnostico

\section{INTRODUÇ̃̃̃O}

Os testes eletrofisiológicos, como eletrorretinografia (ERG) e potencial visual evocado (PVE), são de grande importância clínica sendo amplamente 
utilizados como método diagnóstico, respectivamente para doenças que afetam as estruturas retinianas e para as que afetam as vias ópticas e córtex visual ${ }^{(1-2)}$.

As patologias que afetam as estruturas retinianas podem ser investigadas de maneira mais específica através do ERG. Sendo um dos poucos métodos não invasivo para testar funções elétricas das células da retina, esse exame eletrofisiológico auxilia no diagnóstico, muitas vezes precedendo alterações anatômicas encontradas no exame de fundo de olho, ou é utilizado para um seguimento dessas doenças. As indicações para o ERG incluem sintomas de cegueira noturna, fotofobia, nistagmo e baixa de visão a ser esclarecida. O ERG facilita a distinção entre defeitos funcionais no sistema de cones e bastonetes, entre disfunção de cegueira congênita estacionária e degenerações tapeto-retinianas progressivas. Em algumas doenças retinianas, como acromatopsia, distrofia de cones progressiva e disfunções retinianas por agentes tóxicos, as respostas do ERG apresentam comprometimento antes que alterações fundoscópicas sejam evidenciadas ${ }^{(3)}$. O comprometimento do ERG nos processos inflamatórios da retina, trauma ocular, retinopatia diabética está na dependência da extensão e profundidade do envolvimento retiniano, sendo que tanto nos processos inflamatórios, em geral, como na neurorretinite difusa subaguda unilateral (DUSN), em particular, a onda-b é usualmente mais atingida que a onda-a-a ${ }^{(4-5)}$.

O exame eletrofisiológico de Potencial Visual Evocado reflete a atividade elétrica do córtex visual, em microvolts, em resposta a um estímulo visual, registradas a partir de eletrodos fixados na região suprajacente ao córtex visual, sendo capaz de detectar alterações funcionais em toda a extensão da via máculo-occipital de acordo com o estímulo empregado, assim como a medida objetiva da acuidade visual.

O ERG e o PVE são testes objetivos que independem da informação do paciente para se chegar aos resultados, embora necessitem de certa colaboração para serem executados.

O objetivo deste estudo foi determinar a freqüência de categorias diagnósticas obtidas em serviço de eletrofisiologia visual clínica de hospital universitário.

\section{MÉTODOS}

\section{Participantes}

Foram incluídos neste estudo transversal pacientes submetidos a exames eletrofisiológicos encaminhados para o Laboratório de Eletrofisiologia Visual Clínica do Departamento de Oftalmologia da UNIFESP - Escola Paulista de Medicina, no período de outubro de 1998 a outubro de 2000, num total de 570 pacientes, sendo 320 masculinos e 240 femininos.

O diagnóstico final foi dado considerando-se os achados eletrofisiológicos, comparando-os com normas descritas na literatura $^{(6-7)}$, juntamente com o exame de fundoscopia e outros exames relacionados (angiofluoresceinografia, campo visual, visão de cores).

\section{Eletrorretinografia}

O exame de eletrorretinografia foi feito de acordo com as diretrizes da International Society for Clinical Electrophysiology of Vision (ISCEV) $)^{(1-2)}$. Para a obtenção dos registros do ERG dilatou-se as pupilas do paciente através da instilação de uma gota de tropicamida $1 \%$ e uma gota de fenilefrina $10 \%$, em seguida os olhos foram devidamente ocluídos por 30 minutos para adaptação da retina ao escuro. Após a adaptação ao escuro, foi instilado colírio anestésico (tetracaína 1\%) e um eletrodo de lente de contato bipolar foi colocado sobre a córnea anestesiada, com colírio de metilcelulose a $2 \%$ para proteção da superfície corneana. Um eletrodo terra de cúpula de ouro em forma de clipe, preenchido com gel eletrolítico, foi colocado no lobo da orelha. O paciente foi posicionado numa cúpula de estimulação de campo total (Ganzfeld) e 5 passos do protocolo padrão da ISCEV foram seguidos: resposta de bastonetes, resposta máxima (bastonetes e cones), potenciais oscilatórios, resposta de cones e resposta ao flicker $30 \mathrm{~Hz}^{(2)}$.

O sistema eletrodiagnóstico UTAS E-3000 (LKC Technologies Inc., Gaithesburg, MD, USA) foi utilizado para o registro e análise das ondas do ERG. O ERG de campo total e/ou focal foi realizado em 385 pacientes (68\%).

\section{Potencial Visual Evocado}

O procedimento de potencial visual evocado foi solicitado por dois principais motivos: a avaliação da acuidade visual em pacientes não verbais (bebês, crianças até 3 anos de idade, pacientes com comprometimento neurológico que impeça a comunicação verbal) e para avaliação da integridade da via máculo-occipital. Para avaliação da acuidade visual foi feito o potencial visual evocado de varredura. Nos demais casos foi feito o PVE clássico e por flashes de luz.

O exame de PVE foi feito com dois tipos de estimulação: por reversão de padrões (estímulos estruturados) e por flashes de luz (estímulos não estruturados).

O registro do PVE foi obtido por eletrodos de cúpula de ouro posicionados em $\mathrm{Oz}$ (ativo), $\mathrm{Cz}$ (eletrodo terra) e $\mathrm{FPz}$ (referência), com pasta condutora de sinais elétricos. No PVE clássico, dois tamanhos de estímulos de "tabuleiro de xadrez" foram apresentados, formando respectivamente ângulos visuais de $15^{\prime}$ e $1^{\circ}$, com taxa de reversão de $1,9 \mathrm{~Hz}$. O PVE por flashes de luz foi feito com estimulação na cúpula de campo total, com taxa de reversão de 1,9 Hz. Ambos os exames foram feitos de acordo com o protocolo sugerido pela ISCEV ${ }^{(6)}$.

$\mathrm{O}$ exame de PVE de varredura foi feito em pacientes não verbais, exclusivamente para medida da acuidade visual de resolução de grades. Neste exame, o eletrodo ativo foi posicionado em $\mathrm{Oz}$, a referência a $2 \mathrm{~cm}$ do ativo e o eletrodo terra em $\mathrm{Cz}$ com pasta condutora de sinais elétricos. Foi feita uma varredura de grades de onda quadrada, com contraste de $100 \%$ e taxa de reversão de $6 \mathrm{~Hz}$, com 10 freqüências espaciais crescentes. Após a transformação discreta de Fourier, um algorítmo de extrapolação do pico da amplitude para a amplitude zero por regressão linear foi feito e a acuidade foi estimada. 
O potencial visual evocado por reversão de padrões (clássico ou de varredura) e/ou flashes de luz foi realizado em 185 pacientes (32\%). O sistema UTAS E-3000 (LKC Technologies Inc., Gaithersburg, MD, USA) foi usado para registro e análise das ondas do PVE.

\section{Aspectos Éticos}

Este estudo seguiu as diretrizes da declaração de Helsinque e todos os participantes estavam cientes e consentiram que os dados obtidos em seus exames fossem posteriormente analisados com finalidade de pesquisa.

\section{RESULTADOS}

\section{Eletrorretinografia}

Após avaliação do eletrorretinograma (ERG), as categorias diagnósticas mais freqüentes foram: retinose pigmentária $(\mathrm{N}=$ $110-28,5 \%)$, doença de Stargardt $(\mathrm{N}=23-6,0 \%)$, distrofia de cones $(\mathrm{N}=22-5,7 \%)$, trauma ocular $(\mathrm{N}=19-4,9 \%)$, degeneração do tipo cone-bastonete $(\mathrm{N}=19-4,9 \%)$, retinotoxicidade devido a diferentes agentes (cloroquina, tamoxífeno, metanol) $(\mathrm{N}=17-4,4 \%)$, além de outros diagnósticos $(\mathrm{N}=87-22,5 \%)$. Em $22,8 \%$ dos casos $(\mathrm{N}=88)$, não foi possível obter um diagnóstico final havendo mais de uma hipótese a ser confirmada com a progressão da doença. A figura 1 mostra esses resultados.

Dos 110 casos de retinose pigmentária, 91 foram casos isolados e 19 sindrômicos, sendo 10 com síndrome de Usher, 6 com síndrome de Bardet-Biedl, 1 com síndrome de Kearns-Sayre, 1 com síndrome de Cockayne e 1 com síndrome de Goldenhar.

As outras categorias diagnósticas encontradas em 88 pacientes são listadas na Tabela 1 .

Como demonstrado na figura 1 , em $23 \%$ dos casos $(\mathrm{N}=88)$ não foi possível estabelecer uma categoria diagnóstica, devi-

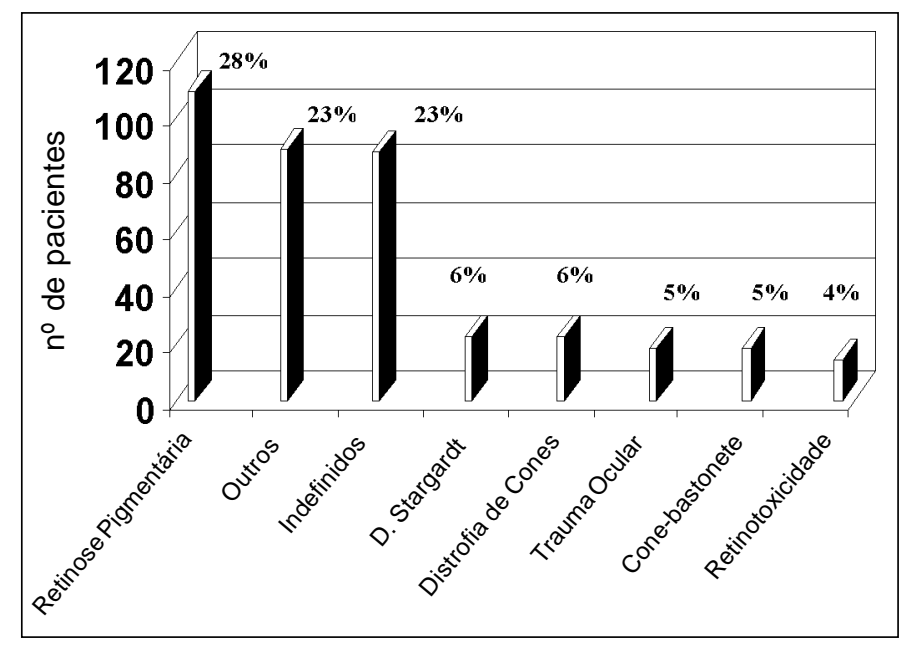

Figura 1 - Freqüência de categorias diagnósticas após avaliação do ERG em 385 pacientes

\begin{tabular}{|lc|}
\hline Tabela 1. Outras categorias diagnósticas de 88 casos após ERG \\
Categoria diagnóstica & Número de casos \\
Degenerações tapeto-retinianas & 30 \\
Doenças do nervo óptico & 16 \\
Uveítes & 13 \\
Ambliopia & 9 \\
Opacidade de meios & 8 \\
Oclusão de veias central da retina & 3 \\
Retinopatia serosa central & 3 \\
Albinismo & 2 \\
Nistagmo congênito & 2 \\
Pseudo buraco de mácula & 1 \\
Simulação de perda visual & 1 \\
Total & 88 \\
\hline
\end{tabular}

do a: dúvida sobre o aspecto de fundo de olho e/ou angiofluoresceinografia; diagnóstico diferencial entre distrofia de cones e degeneração cone-bastonete, doenças genéticas que estão sob investigação e nas maculopatias. Além disso, houve casos em que o exame de eletrorretinografia foi solicitado como exame complementar em pacientes com queixas visuais, mas com acuidade visual normal para idade e resultado do ERG normal.

\section{Potencial Visual Evocado}

O PVE para avaliação da integridade da via máculo-occiptal foi realizado em 101 pacientes $(54,6 \%)$. As causas mais freqüentes foram doenças neurológicas $(\mathrm{N}=32)$, seguidas por doenças que afetam o nervo óptico $(\mathrm{N}=29)$, opacidade de meios $(\mathrm{N}=17)$ e outras $(\mathrm{N}=15)$. Em 7 casos não foi possível chegar a um diagnóstico final após o PVE.

A tabela 2 mostra os resultados das categorias diagnósticas para o PVE clássico.

O PVE de varredura foi feito em 84 pacientes $(45,4 \%)$, sendo as condições mais freqüentes a catarata congênita $(\mathrm{N}=$ 32 ), seguida por glaucoma congênito $(\mathrm{N}=14)$, deficiência visual cortical $(\mathrm{N}=10)$, doenças genéticas $(\mathrm{N}=10-$ síndrome de Peters, síndrome de Morquio, síndrome de Lowe, síndrome de Freeman-Sheldon, síndrome de Moebius, amaurose congênita de Leber, etc.), retinoblastoma $(\mathrm{N}=5)$, retinopatia da prematuridade $(\mathrm{N}=3)$, estrabismo $(\mathrm{N}=2)$ e nistagmo $(\mathrm{N}=2)$. Outros diagnósticos com 1 caso em cada categoria foram: maturação visual atrasada, síndrome fetal alcóolica, síndrome de West, doença de Tay-Sachs, anoftalmia e microftalmia.

\section{DISCUSSÃO}

A investigação eletrofisiológica pelo ERG e pelo PVE tem sido amplamente utilizada como método diagnóstico complementar em distúrbios que afetam a retina e/ou a via visual ${ }^{(8-9)}$.

Como descrito anteriormente, em 7 casos não foi possível chegar a um diagnóstico final após o PVE, devido à falta de colaboração do paciente para a realização do exame.

Em 1968, Halter e White, ao simularem ametropias em in- 


\begin{tabular}{|c|c|}
\hline \multicolumn{2}{|c|}{ Tabela 2. Diagnósticos de 94 casos após exame de PVE } \\
\hline \multicolumn{2}{|c|}{$\begin{array}{l}\text { Condições neurológicas que afetam a via visual }(\mathrm{N}=32) \\
\text { Categoria diagnóstica }\end{array}$} \\
\hline Esclerose múltipla & 8 \\
\hline Paralisia cerebral & 6 \\
\hline Trauma crânio-encefálico & 3 \\
\hline Epilepsia & 2 \\
\hline Neurocisticercose & 2 \\
\hline Outros* & 11 \\
\hline Subtotal & 32 \\
\hline \multicolumn{2}{|c|}{ Doenças que afetam o nervo óptico $(\mathrm{N}=29)$} \\
\hline Atrofia de nervo óptico & 14 \\
\hline Neurite óptica & 4 \\
\hline Neuropatia óptica & 3 \\
\hline Síndrome tabaco-álcool & 2 \\
\hline Outros $^{* *}$ & 6 \\
\hline Subtotal & 29 \\
\hline $\begin{array}{l}\text { Opacidade de meios }(\mathrm{N}=18) \\
\text { Categoria diagnóstica }\end{array}$ & Número de Casos \\
\hline Trauma ocular & 7 \\
\hline Leucoma & 5 \\
\hline Esclerocórnea & 2 \\
\hline Outros $^{* * *}$ & 4 \\
\hline Subtotal & 18 \\
\hline \multicolumn{2}{|c|}{ Outras categorias diagnósticas $(\mathrm{N}=15)$} \\
\hline Uveítes & 8 \\
\hline Simulação de baixa de visão & 2 \\
\hline Retinopatia diabética & 2 \\
\hline Retinopatia serosa central & 1 \\
\hline Toxicidade por cloroquina & 1 \\
\hline Doença de Lyme & 1 \\
\hline Subtotal & 15 \\
\hline Total Geral & 94 \\
\hline \multicolumn{2}{|c|}{$\begin{array}{l}\text { *lesão cerebral pós PCR, aneurisma cerebral, anóxia, atrofia cerebelar, } \\
\text { acidente vascular cerebral, esquisencefalia, hidrocefalia, microcefalia, } \\
\text { neurofibromatose, tumor cerebelar, encefalopatia. } \\
\text { **edema de disco, neuropatia tóxica, hipoplasia de nervo óptico, coloboma de } \\
\text { nervo óptico, glaucoma }\end{array}$} \\
\hline
\end{tabular}

divíduos emétropes, observaram a diminuição da amplitude de determinados componentes do PVE. Por isso, para o exame de potencial visual evocado, quando feito com estímulos estruturados, que consiste em estímulos formados por padrões como quadrados e barras, exibindo forma, contorno e contraste, o paciente foi examinado com sua ametropia corrigida, quando necessário.

A International Society for Clinical Electrophysiology of Vision recomenda o estabelecimento de valores normativos para cada laboratório de eletrofisiologia visual clínica, como o estudo normativo deste laboratório estava sendo feito paralelamente a este, os resultados dos registros foram analisados de acordo com normas descritas na literatura ${ }^{(6-7)}$.

Os resultados apresentados corroboram achados de estudos anteriores sobre a freqüência de categorias diagnósticas mais encontradas após registro do $\mathrm{ERG}^{(10)}$. As indicações mais freqüentes para o registro eletrofisiológico visual encontradas neste grupo relativamente grande de pacientes estudados, confirmam a contribuição fundamental destes testes para decisões diagnósticas. Diagnósticos como estágios iniciais da retinose pigmentária, a distrofia progressiva de cones, a retinopatia tóxica sem alterações fundoscópicas, a disfunção de retina ou de nervo óptico na presença de meios opacos, o envolvimento do nervo óptico na esclerose múltipla puderam ser concluídos após a avaliação eletrofisiológica. Além disso, a medida da acuidade visual em condições oculares pediátricas e/ou neurológicas, nos pacientes que não podem informar a acuidade pode ser obtida.

\section{CONCLUSÃO}

Após análise deste amplo grupo de pacientes, ficam evidentes as indicações mais freqüentes e significativas para registros eletrofisiológicos visuais e sua contribuição em decisões diagnósticas. Vários diagnósticos podem ser de difícil conclusão sem os testes eletrofisiológicos visuais. Dentre essas patologias incluem-se estágios iniciais da retinose pigmentária, distrofia progressiva de cones, retinopatia tóxica sem alterações fundoscópicas, disfunção de retina ou de nervo óptico na presença de meios opacos, envolvimento de nervo óptico em esclerose múltipla e avaliação da acuidade visual em doenças oculares infantis com ou sem alterações neurológicas.

\section{AGRADECIMENTOS}

Agradecemos o apoio financeiro recebido pela FAPESP (processo 97/11493-3) à Solange Rios Salomão para a aquisição do equipamento utilizado neste estudo.

\section{ABSTRACT}

Purpose: To determine the frequency of diagnostic categories obtained in a clinical vision electrophysiology laboratory at a university hospital. Methods: Patients who underwent visual electrodiagnostic testing from October 1998 to October 2000 $(\mathrm{N}=570)$ were cross-sectionally studied. Electroretinogram (ERG) was performed in 385 (68\%) patients and pattern and/or flash visually evoked potentials were performed in $185(32 \%)$ patients. Results: After electroretinogram assessment, the most frequently identified diagnostic categories were (in this order): retinitis pigmentosa, Stargardt's disease, cone dystrophy, cone-rod degeneration, retinotoxic effects due to several agents (chloroquine, tamoxifen, methanol, etc) and ocular trauma, besides unclear visual loss. In $23 \%$ of the cases, it was impossible to obtain a final diagnosis on the first examination. The largest group referred to electroretinogram testing was for documentation or exclusion of tapetoretinal degenerations (42\%). In this group, 110 cases of RP were identified: 91 isolated and 19 syndromic cases. Visually evoked potential testing helped to identify the following diagnostic categories: diseases affecting only the optic nerve (optic nerve atrophy, 
optic neuritis, optic disk edema, etc); neurological conditions affecting the visual pathway (multiple sclerosis, cerebral palsy, blunt head trauma, tumors, etc): pediatric eye and/or neurological conditions (congenital cataract, cortical visual impairment, congenital glaucoma, etc) and opaque media (leucoma, ocular trauma, etc). Conclusions: Frequent and meaningful indications for visual electrophysiologic recording and diagnostic decisions arise from this relatively large group of studied patients. A number of diagnoses can hardly, if not all, be established without visual electrophysiologic testing. These include early stages of retinitis pigmentosa, progressive cone dystrophy, toxic retinopathy without fundus changes, optic nerve or retinal dysfunction in opaque media, optic nerve involvement in multiple sclerosis and pediatric eye disease with or without neurological damage.

Keywords: Electroretinography; Retina/physiopathology; Visual evoked potentials; Vision/physiology; Electrophysiology/methods; Vision tests; Retinal diseases/diagnosis; Retinitis pigmentosa/diagnosis

\section{REFERÊNCIAS}

1. Paranhos FRL, Ávila MP, Paranhos A, Cialdini AP. Estudo estatísticos de valores normais do eletrorretinograma: contribuição à padronização do exame. Arq Bras Oftalmol 1997;60:278-84.

2. Standard for clinical electroretinography. International Standardization Committee. Arch Ophthamol 1989;107:816-9.

3. Niemeyer G. From the symptom to electroretinography diagnosis. Klin Monatsbl Augenheilkd 1999;214:328-31.

4. Gonçalves ER, Oréfice F. Eletrofisiologia nas uveítes. In: Oréfice F, Belfort Jr R. Uveítes. São Paulo: Rocca; 1987. p.116-40.

5. Oréfice F, Gonçalves ER, Siqueira RC, Nehemy MB. Estudo de 21 casos de neurorretinite subaguda unilateral difusa (DUSN); dois casos com larva móvel sub-retiniana. Rev Bras Oftalmol 1994;53:467-89.

6. Harding FA, Odom JV, Spileers W, Spekreijsp H. Standard for visual evoked potentials 1995. The International Society for Clinical Electrophysiology of Vision. Vision Res 1996;36:3567-72.

7. Clinch TE, Kaufman HE. Standardized full-field electrorretinography. Normal values and their variation with age. Arch Ophthalmol 1992;110:1571-6.

8. Paranhos FRL, Nehemy MB, Hirose T. Bilateridade, simetria e correlação clinico-eletrofisiológica na retinose pigmentar. Arq Bras Oftalmol 1998;61:386-9.

9. Sibinelli MMF, Cohen RR, Tilbery AM, Lake CP, Jonathan C. Manifestações oculares em pacientes com esclerose múltipla em São Paulo. Arq Bras Oftalmol 2000;63:287-91.

10. Stemeyer G, Stähli P. ERG diagnosis and differential diagnosis: results of examination over 6 years. Klin Monatsbl Augenheilkd 1996;208:306-10.

\section{Curso Avançado de Retina, Uveítes e Tumores}

\section{5 a 28 de Junho de 2003}

\section{Hotel Caesar Park - Fortaleza - CE}

\section{Promoção:}

Sociedade Brasileira de Retina e Vítreo

Centro Especializado de Retina e Vítreo

INFORMAÇÕES: Tels.: (85) 272-1572 / 272-7795

Home page: www.accvia.com.br 